\title{
Situación y perspectivas de la investigación científica en la Escuela de Bibliotecología y Ciencias de la Información de la Universidad Nacional Mayor de San Marcos: 2000-

Alonso Estrada-Cuzcano

Docente de la Escuela de Bibliotecología y Ciencias de la Información. Facultad de Letras y Ciencias Humanas daUniversidad Nacional Mayor de San Marcos (Lima, Perú)

Karen Lizeth Alfaro-Mendives

Facultad de Letras y Ciencias Humanas da Universidad Nacional Mayor de San Marcos (Lima, Perú)

http://dx.org/10.1590/1981-5344/1933

Se analiza el ascenso en la cantidad de egresados de Bibliotecología que optan por la licenciatura a través de informes profesionales en los últimos años. Asimismo, se pretende conocer el estado de la investigación en bibliotecología en la Universidad Nacional Mayor de San Marcos a través del análisis de las tesis e informes profesionales de los años 2000 - 2012, determinando la cantidad de investigación producida, los temas abordados, métodos y técnicas de investigación más utilizadas, cual es la calidad investigativa de estos trabajos. Se propone como se debería reorientar la investigación de los egresados de la especialidad para la obtención de su licenciatura.

Palabras claves: Investigación bibliotecológica; Escuela de Bibliotecología y Ciencias de la Información; Universidad Nacional Mayor de San Marcos. 


\section{Situation and prospects of scientific research in the School of Library and Information Sciences in National University of San Marcos: 2000-2012}

It analyzes the rise in the number of graduates who choose Librarianship degree through professional reports in recent years. It also seeks to know the status of research in library science at the National University of San Marcos through the analysis of theses and professional reports of the years 2000 - 2012, determining the amount of research produced, the topics, methods and research techniques used, which is the investigative quality of this work. It is proposed as research should reorient specialty graduates to obtain their degree.

Key-words: Library Research; School of Library and Information Sciences; National University of San Marcos.

\section{Situação e perspectivas de pesquisa científica na Escola de Biblioteconomia e Ciência da Informação da Universidade Nacional Maior de São Marcos: 2000 - 2012}

É analisado o aumento do número de diplomados que optam pelo grau de Biblioteconomia nos últimos anos através de relatórios profissionais. Pretende conhecer 0 estado da pesquisa em biblioteconomia na Universidade Nacional Maior de São Marcos, através da análise de teses e relatórios profissionais para os anos 2000 - 2012, para determinar a quantidade de pesquisas feitas, os seus asuntos, os principais métodos e técnicas de pesquisa utilizadas, assim como qual é a qualidade de pesquisa destes trabalhos. Em consequência, propõe-se como se deve redirecionar aos graduados da especialidade de pesquisa para obter o seu grau. 
Palavras chave: Pesquisa em Biblioteconomia; Escola de Biblioteconomia - Ciência da Informação; Universidade Nacional Maior de São Marcos

Recebido em 27.11.2013 Aceito em 10.04.2014

\section{Descripción del problema}

La Universidad Nacional Mayor de San Marcos (UNMSM) es el primer centro de estudios fundado en América (12 de mayo de 1551) por Real Cédula firmada por el Rey Carlos $V$ en la ciudad de Valladolid, y se le dio por nombre Universidad de Lima. Es a partir de 1574 que toma el nombre de Universidad de San Marcos. La Facultad de Letras fue una de las primeras facultades que funciono en el claustro.

Precisamente la Escuela Académico Profesional de Bibliotecología y Ciencias de la Información (EAPBCI) obtiene rango universitario en 1981 y actualmente depende de la Facultad de Letras y Ciencias Humanas (tiene otras 7 escuelas: Comunicación Social, Literatura, Lingüística, Arte, Conservación y Restauración, Filosofía y Danza); la EAPCI tiene hasta la fecha un aproximado de 300 alumnos.

Actualmente, se observa que la gran mayoría de los egresados de la EAPBCI prefieren optar por la modalidad de informe profesional antes que la elaboración de tesis. Si bien es cierto los informes profesionales ameritan un cierto nivel de investigación a nivel exploratorio y descriptivo, y en algunas veces se requiere realizar un trabajo de campo y la aplicación de un instrumento, son básicamente propuestas para solucionar un problema de tipo laboral encontrado por el aspirante y no son investigaciones propiamente dichas, no cuentan con la exhaustividad y rigurosidad académica de una tesis (contrastación de hipótesis, validación de instrumentos, trabajo de campo, análisis estadístico de resultados, etc.). Sin embargo, cabe mencionar, que la rigurosidad metodológica ha mejorado con el transcurso de los años, así como la cantidad de los mismos, ya que cada vez más es la cantidad de graduados en la especialidad en comparación con otras escuelas, pero no se tienen estadísticas formales de qué temas son los que más se tratan actualmente, que métodos y técnicas de investigación utilizan y si las propuestas que se plantean en estos trabajos se llevan a cabo.

Esto debido a que no se cuenta con políticas de fomento a la investigación y sistemas de control y monitoreo de la producción investigativa de los egresados, sin mencionar libros, artículos de revistas, entre otros, claro que esto no forma parte del presente estudio, pero es necesario mencionarlo. El presente trabajo se enfoca exclusivamente al análisis de las tesis e informes profesionales de los egresados de Bibliotecología de la UNMSM en los años 2000 al 2012.

Si ésta situación persiste seguirá esta tendencia a la mínima elaboración de tesis, se realizarán temas aislados que no darán ningún aporte teórico o práctico a la especialidad $y$, mucho peor, serán trabajos 
que solo irán a engrosar los anaqueles de la biblioteca de la Facultad sin ningún aporte. Es por eso que, en el presente estudio se analizará la distribución temática de los informes profesionales, los tipos de unidades de información donde se han realizado estos trabajos, alcance de la investigación, técnicas y métodos utilizados y si se han llevado a cabo las propuestas, a efectos de proponer lineamientos de mejora para reorientar la investigación en Bibliotecología en la UNMSM.

\section{Antecedentes}

Se muestran los trabajos que reflejan la producción científica de la UNMSM, Peña et al (2011) ordenan y recogen cifras de la actividad investigadora de los docentes por áreas a partir de la base de datos Web of Knowledge de ISI integrada a Thompson Reuters; del mismo libro extraemos un capítulo elaborado por Estrada-Cuzcano et al. (2011) que centran su análisis en la producción científica de los docentes de la Facultad de Letras y Ciencias Humanas de la UNMSM y determinan a través del examen de diversas bases de datos científicas (comerciales y Open Access) la visibilidad de los trabajos evaluados.

Hay pocos antecedentes que muestren la evolución de la producción científica en de la EAPBCI pero señala Bibliotecología; el primer trabajo que recopila tesis de la antigua Escuela Nacional de Bibliotecarios (antecesora de la EAPBCI) pertenece a Silva Santisteban Cevallos (1984); posteriormente la Revista Claustro Pleno hace un inventario del universo de publicaciones peruanas sobre Bibliotecología (Corzo et al., 1986). La Biblioteca Nacional del Perú realizó dos encuentros de investigadores el primero en el año 2002 (BNP, 2003) y en el año 2007 (BNP, 2009) en los que se debatió primordialmente la situación y características de la investigación, pero no se hace un análisis de la producción científica; de esos encuentros tomamos los trabajos de Corzo C. (2002) quien describe de forma cuantitativa (número y área de estudio) las tesis presentadas en las Escuelas de Bibliotecología existentes en el Perú; mientras que Quispe Gerónimo (2009) presenta un trabajo bibliométrico sobre la investigación en Bibliotecología publicada por autores peruanos en E-LIS (E-prints in Library \& Information Science). Finalmente Vílchez Román (2005) publicó un informe basado en una encuesta aplicada a bibliotecarios para determinar las características de las investigaciones publicadas; así como, el alcance, situación, apoyo económico, temas, métodos de investigación utilizados, entre otros indicadores.

Hay artículos referidos al estado del arte de la EAPBCI pertenecientes a Quiroz de García (2002) y Miranda Meruvia (2005); en ambos se puede establecer la evolución de los grados y títulos aunque no se examina la producción científica.

\section{La investigación en la UNMSM}

El Vicerrectorado de Investigación fue creado el año 2005 y tiene como visión "Ser referente nacional e internacional en la investigación 
científica, humanística e innovadora". Este Vicerrectorado ha planteado una política de investigación dentro de la universidad; existen incentivos a la investigación científica, actualmente se subvencionan proyectos de investigación y se incluye a la Facultad de Letras y Ciencias Humanas otorgándole 33 subvenciones anualmente. También hay subvenciones para elaboración de tesis de pregrado y se otorgan a 10 trabajos de tesis de estudiantes de la Facultad cada año. La Facultad además promociona talleres de investigación anuales que congrega a todas especialidades y es un entorno ideal para discutir nuevas tendencias en la investigación.

La Facultad de Letras y Ciencias Humanas, tiene una Unidad de Investigación que coordina a cuatro institutos de investigación, que a continuación mencionamos: IPPLA: Instituto del Pensamiento Peruano y Latinoamericano; CILA: Centro de Investigación de Lingüística Aplicada); IIH: Instituto de Investigaciones Humanísticas y el INVEL: Instituto de Investigaciones Lingüísticas.

Es preciso decir que La UNMSM ocupa el segundo lugar en producción científica en el Perú aunque las cifras son muy modestas tal como podemos ver en el Gráfico 1 y podemos ver la evolución en los últimos años.

Gráfico 1

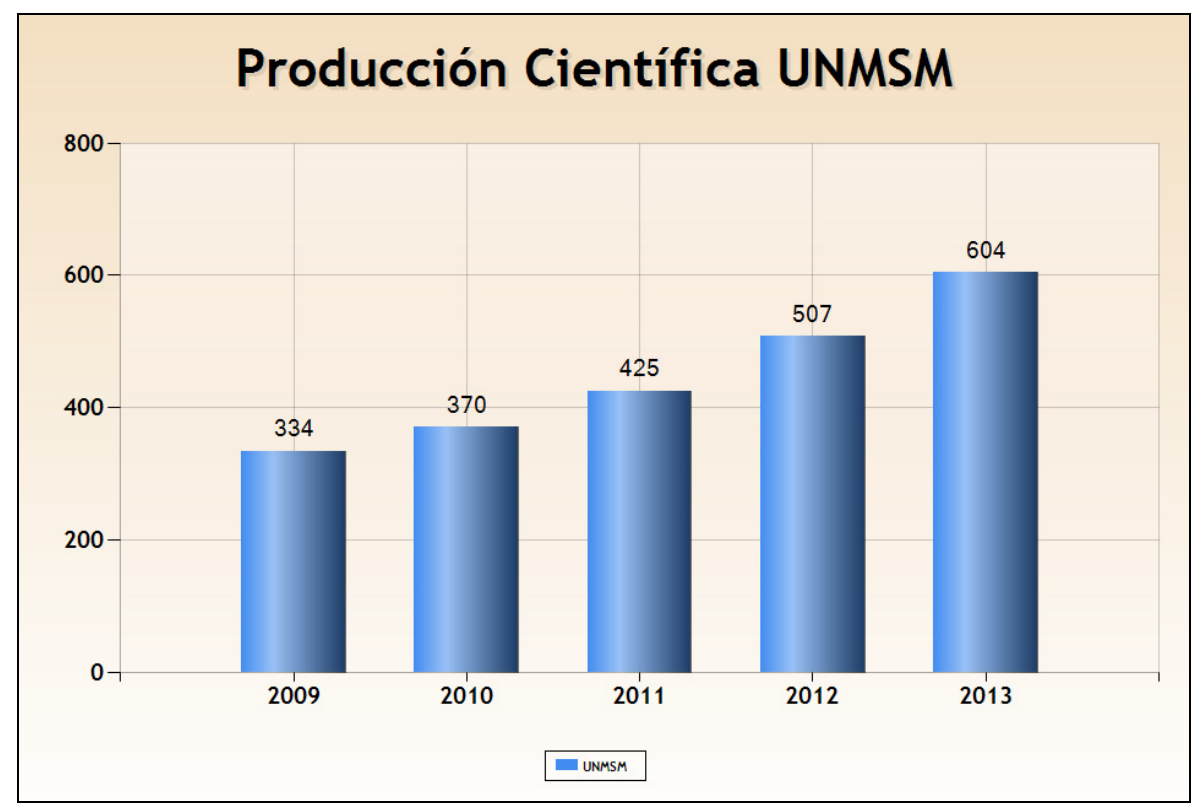

Fuente: SIR Iberoamericano (Scimago)

\section{$4 \quad$ Análisis de las tesis de la EAPBCI de la UNMSM}

Actualmente existen dos modalidades de titulación; la primera es la presentación de una tesis y la segunda es la presentación de un informe profesional (experiencia de campo). El número de tesis que se presentan para la obtención del título profesional es mínimo; mientras la presentación de informes profesionales aumenta continuamente; razón 
por la cual es necesario conocer la rigurosidad metodológica y alcance investigativo de los mismos.

Asimismo, el presente estudio servirá de base para quienes quieran ahondar sobre las tendencias de investigación en Bibliotecología y Ciencias de la Información que tienen como principal elemento a la investigación universitaria (tesis e informes). Además, se proponen lineamientos para mejorar el nivel investigativo de la especialidad en la UNMSM y deberían tomarse en consideración por los estudiantes y la propia Escuela.

Los objetivos trazados son:

\section{Objetivo general}

Determinar la situación actual de la investigación en la Escuela de Bibliotecología de la Universidad Nacional Mayor de San Marcos en el periodo 2000 al 2012.

\section{Objetivos específicos}

Identificar los tipos de unidades y áreas de la especialidad donde se ha realizado el mayor número de investigaciones.

Analizar el alcance metodológico, las técnicas e instrumentos de recolección de la información utilizado en los trabajos de investigación.

Determinar en qué medida las propuestas planteadas en cada una de las investigaciones tienen aplicación práctica.

Proponer lineamientos para reorientar la investigación en la Escuela de Bibliotecología y Ciencias de la Información.

\section{$5 \quad$ Metodología}

\section{a) Tipo de investigación}

La investigación es de tipo descriptivo, mostrará cual es la situación actual de la investigación, las áreas de la especialidad donde más se investiga, el alcance de las investigaciones y si se han llevado a cabo las propuestas en la práctica que realizan los egresados de la Escuela de Bibliotecología de la UNMSM.

\section{b) Instrumento de recolección de datos}

Se analizaron las tesis y los informes profesionales producidos a partir del año 2000 al 2012 registrados en la Biblioteca de la Facultad de Letras y Ciencias Humanas de la UNMSM, siendo en total 261 trabajos, para evitar el margen de error se estudió la totalidad de los trabajos realizados.

Se aplicó a cada trabajo la guía de registro de informes profesionales que contenía información como: título, año, área de investigación, tipo de unidad de información, propuesta e instrumento de recolección de la información. Asimismo, se aplicó una encuesta con cuatro (4) preguntas cerradas de opción múltiple, que fue distribuida por correo electrónico del 6 al 13 de julio de 2009 y 20 de octubre al 5 de noviembre de 2013 a cada uno de los egresados para obtener la información necesaria que no se podía obtener con solo la revisión de los informes, como técnica e instrumento de recolección de la información, 
alcance de la investigación realizada (exploratorio, descriptivo, explicativo e histórico), la cual tuvo un porcentaje alto de respuesta debido a la simplicidad de la misma. De 261 egresados, 189 contestaron el instrumento.

Para poder localizar a cada uno de los egresados se intentó ubicar su teléfono y correo electrónico a través del Programa SCU (Sistema de Control de Usuarios de la UNMSM), base de datos de la Escuela de los cursos de Seminario de Actualización y a través de la página del Colegio de Bibliotecólogos del Perú.

c) Análisis e interpretación de los resultados

De la encuesta aplicada estos fueron los resultados:

Es cierto que los datos demográficos no son el objetivo del estudio pero hay algunos datos relevantes. Por ejemplo, en cuanto a la variable sexo, si bien es cierto no es el objetivo del trabajo, es un dato importante, la mayoría de los titulados en Bibliotecología en la UNMSM han sido mujeres en una proporción de 3 a 1 aproximadamente: 197 mujeres (75\% aproximadamente) y 64 hombres (25\% aproximadamente).

La actual ley universitaria permite dos modalidades de titulación: la tesis y el informe profesional; En lo que respecta a la modalidad de titulación, como se observa en la siguiente tabla, los resultados son contundentes, de los 261 trabajos que se han realizado del 2000 al 2012, solo 5 son tesis $(2 \%)$, lo que índica definitivamente la preferencia por la elaboración de informes profesionales (98\%), debido a la facilidad de los mismos y a la rapidez en la obtención del título a través de esta modalidad.

\section{Gráfico 2}

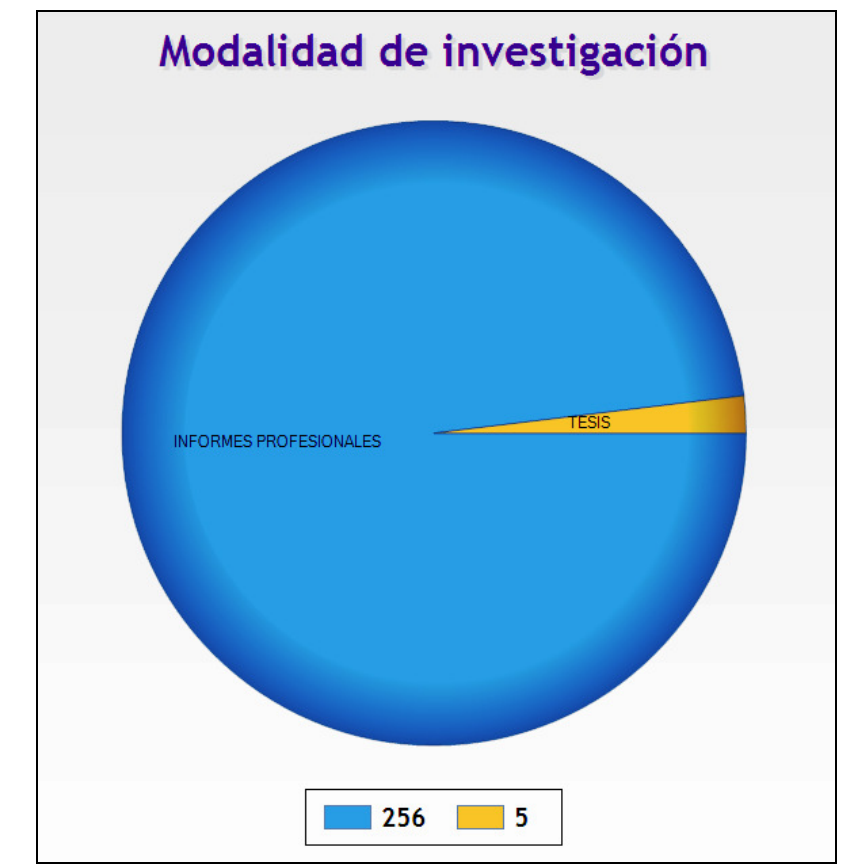

Fuente: Elaboración propia 
Se realizó una comparación de la cantidad de informes por año, como se ha mencionado anteriormente, la presentación de informes ha ido en aumento con una clara tendencia a crecer (GRÁFICO 3). Específicamente a partir del año 2009 (68 informes, $26.1 \%$ aproximadamente) seguido del año 2010 (45 informes, $17.2 \%$ aproximadamente) se ha mantenido una constante (2011 con 35 informes, 13.4\% aproximadamente y 2012 con 34, 13\% aproximadamente) esto se debe básicamente al Seminario de Actualización para la obtención del título profesional que brinda la Escuela de Bibliotecología todos los años, así como la rigurosidad en el cumplimiento de los plazos que no deben sobrepasar de un año; los siguientes años. Las cifras más bajas en la presentación de informes corresponde a los años 2000, 2001 y 2004 (2.7\% aproximadamente por año).

\section{Gráfico 3}

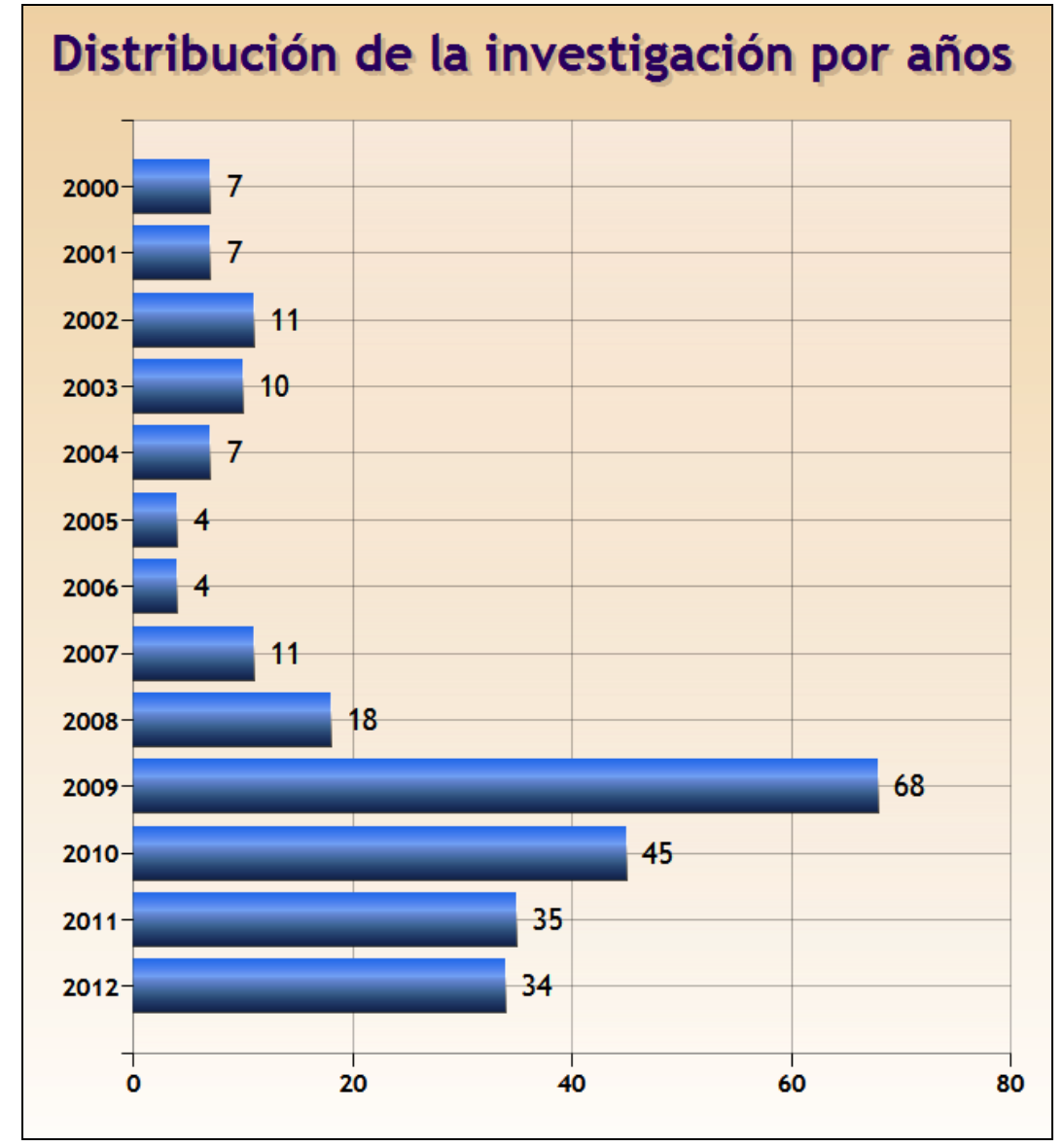

Fuente: Elaboración propia

Se ha realizado una tabla de distribución temática para determinar en qué áreas son las que más están investigando los egresados, como se puede apreciar el tema que más investigan preponderantemente es Servicios Bibliotecarios, seguido de lejos por Administración de Unidades de Información y Evaluación de Unidades de Información. Asimismo, se puede observar que nadie realiza trabajos acerca de la Historia del Libro o 
de la Profesión y casi nadie se aboca a investigar acerca de Derecho o Legislación Bibliotecológica y Bibliometria, esto puede obedecer, básicamente, a que los egresados consideran estos temas poco prácticos en su actividad laboral inmediata.

Tabla 1 Distribución de áreas temáticas de los Informes Profesionales

\begin{tabular}{|l|c|c|}
\hline \multicolumn{1}{|c|}{ Áreas temáticas } & Cantidad & Porcentaje (\%) \\
\hline Servicios bibliotecarios & 58 & $22 \%$ \\
\hline Administración de Unidades de Información & 31 & $12 \%$ \\
\hline Evaluación de unidades de información & 23 & $9 \%$ \\
\hline Procesamiento técnico & 19 & $7 \%$ \\
\hline Archivística y archivos & 17 & $7 \%$ \\
\hline Libros y Hábitos de lectura & 16 & $6 \%$ \\
\hline Desarrollo de colecciones & 15 & $6 \%$ \\
\hline Usuarios de la información & 12 & $5 \%$ \\
\hline Tecnología de la información & 9 & $3 \%$ \\
\hline Conservación y restauración & 7 & $3 \%$ \\
\hline Marketing en unidades de información & 6 & $2 \%$ \\
\hline Acceso y transparencia a la información & 5 & $2 \%$ \\
\hline Infraestructura & 5 & $5 \%$ \\
\hline Repositorios digitales & 4 & $2 \%$ \\
\hline Usabilidad y visibilidad & 4 & $2 \%$ \\
\hline Formación profesional & 3 & $1 \%$ \\
\hline Habilidades informacionales & 3 & $1 \%$ \\
\hline Redes de información & 3 & $1 \%$ \\
\hline Bibliometría & 2 & $1 \%$ \\
\hline Gestión del conocimiento & 2 & $1 \%$ \\
\hline Legislación bibliotecológica & 2 & $1 \%$ \\
\hline Normas técnicas & 2 & $1 \%$ \\
\hline Riesgos en el trabajo & $\mathbf{2 6 1}$ & $\mathbf{2}$ \\
\hline Otros & & $5 \%$ \\
\hline & $\mathbf{1 0 0} \%$ \\
\hline Fuente: Ela & & \\
\hline
\end{tabular}

Fuente: Elaboración propia

En lo que concierne a los tipos de unidades de información estudiadas son contundentes los estudios en mayor cantidad referentes a bibliotecas universitarias (86 informes, 33\% aproximadamente), esto se puede deber a que es el tipo de unidad de información en donde ha habido una evolución importante y que posee el mayor número de egresados, teniendo en cuenta aspectos de evaluación y acreditación que son temas de actualidad; coincidentemente la reducida producción científica peruana en Bibliotecología se centran en este tema, un artículo indexado (Estrada-Cuzcano and Huaman-Huriarte, 2010) y una tesis de pregrado (ALFARO JIMENEZ, 2011). Los estudios en bibliotecas 
especializadas (48 informes, 18\%) aproximadamente se ubican entre los informes más realizados por los egresados, seguido por la Biblioteca Nacional (28 informes, 11\% aproximadamente) y los Archivos (27 informes, $10 \%$ aproximadamente), este último aspecto importante a considerar como un indicador valido del mercado laboral (los informes sistematizan experiencias profesionales). Siguen en orden decreciente las bibliotecas escolares (19 informes, 7\% aproximadamente); centros de documentación (14 informes, 5\% aproximadamente); bibliotecas virtuales (11 informes, 4\% aproximadamente) y bibliotecas públicas (10 informes, $4 \%$ aproximadamente). En este periodo se han realizado pocos estudios aplicados a bibliotecas infantiles (2 informes, 1\% aproximadamente), situación necesaria de considerar porque que estas bibliotecas son el primer contacto del usuario con unidades de información $y$, en complemento con la educación, es un campo interesante y rico para desarrollar.

Gráfico 4

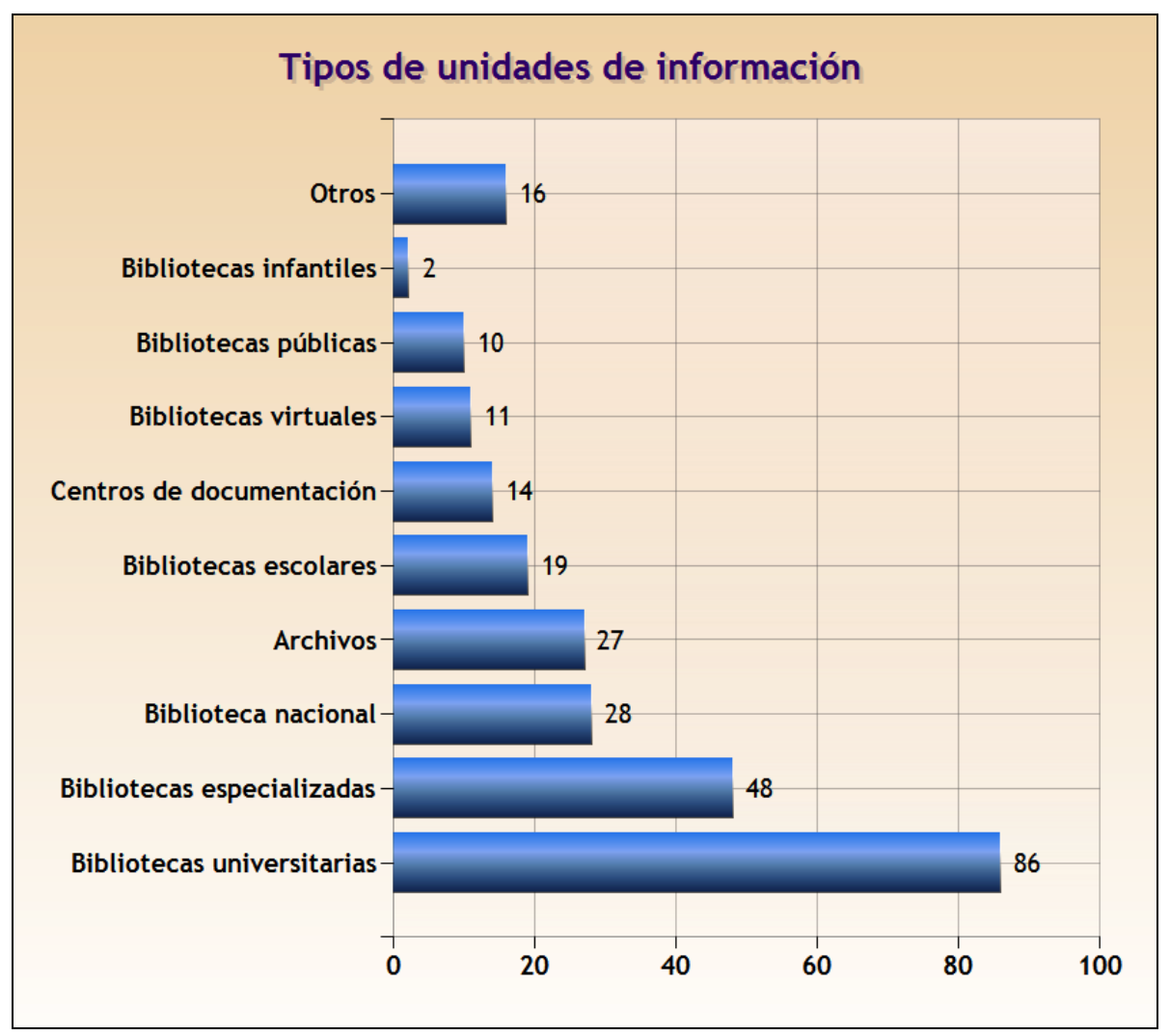

Fuente: Elaboración propia

Lo más importante o el fin primordial de cualquier investigación es el aporte para la especialidad, por esta razón es importante conocer si las propuestas que Se verificó si en cada uno de los informes profesionales se llevaron a cabo en la práctica, como se puede ver en el Gráfico 5, que se muestra a continuación, el $64 \%$ de las propuestas se han cumplido en alguna medida. También es importante mencionar que un $21 \%$ de los trabajos solo sirvieron para la obtención de la licenciatura y pasaron al anaquel sin ningún aporte. Solo un $14 \%$ ha llevado a cabo su propuesta 
en una unidad de información real, aspecto importante a considerar en el futuro porque cuando se realiza una investigación para la obtención de un grado o para la licenciatura existe una doble satisfacción para el aspirante la consecución del título y el aporte a la especialidad, ya sea teórico o práctico.

\section{Gráfico 5}

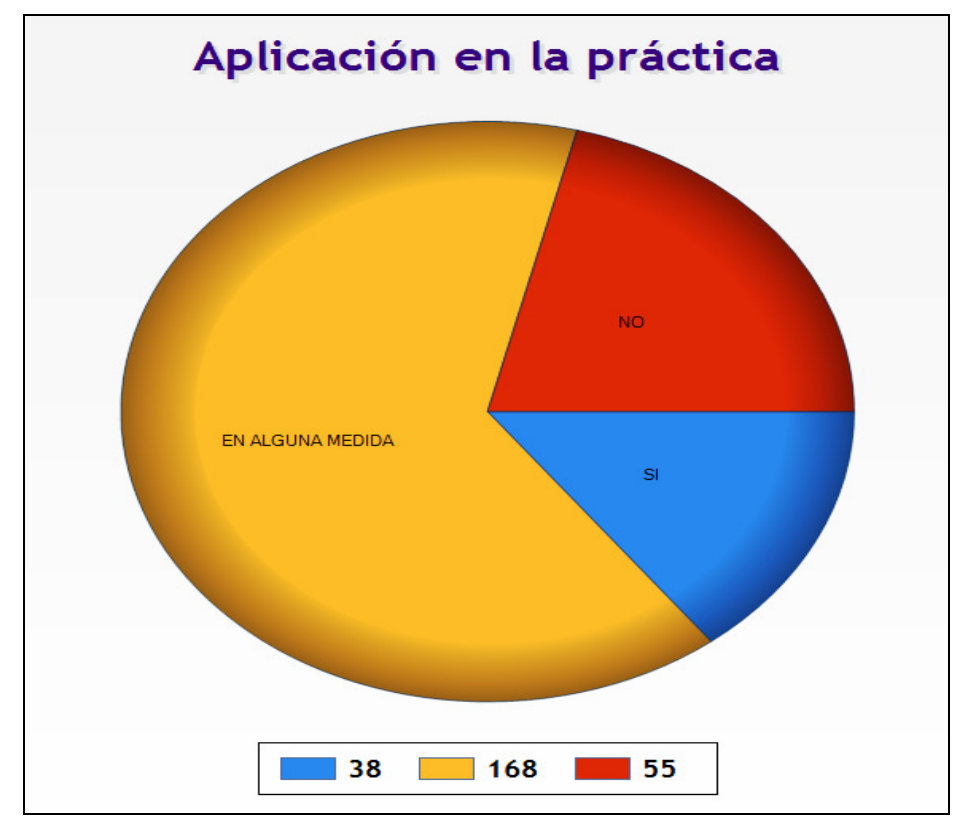

Fuente: Elaboración propia

En cuanto al alcance de la investigación, en su gran mayoría informes profesionales son básicamente trabajos descriptivos (241 informes, $92 \%$ aproximadamente), que básicamente identifican características, propiedades establecer conductas y comportamientos, dirigidos a establecer el cómo y no por el porqué. Es interesante notar que son mínimos los trabajos históricos (2 informes, $0.77 \%$ aproximadamente), línea de investigación que se debe implementar con urgencia, necesaria también, para el desarrollo de cualquier especialidad.

\section{Gráfico 6}

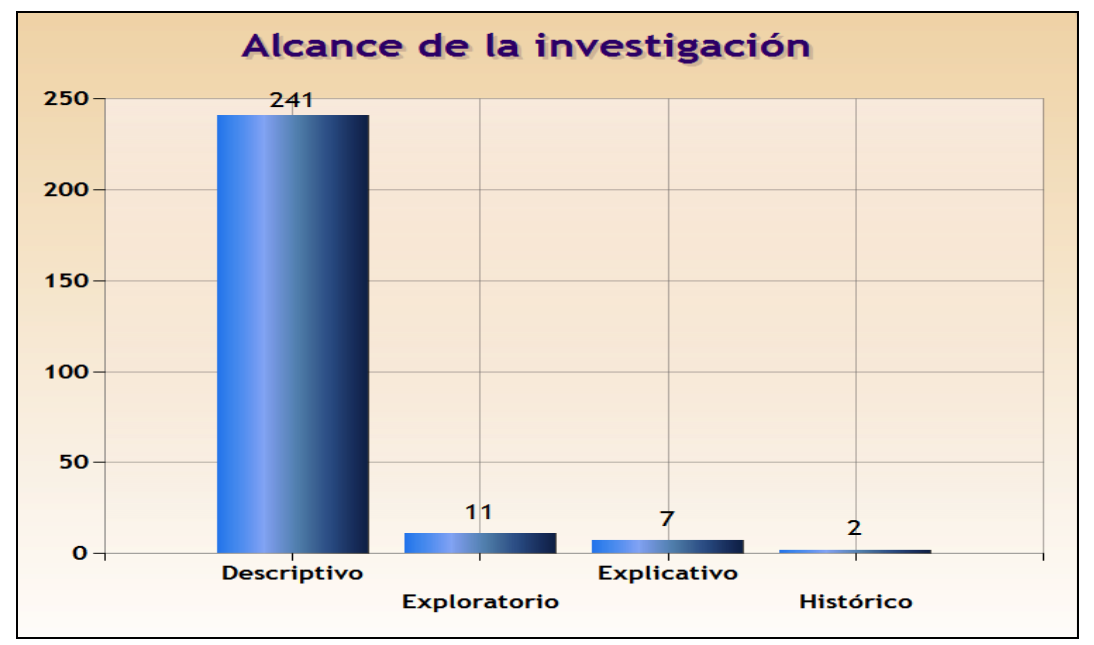


Fuente: Elaboración propia

Con respecto a la técnicas de investigación, los resultados fueron mayor al número de la muestra (275), debido a que muchos de los encuestados respondieron que en sus investigaciones utilizaron más de 1 (una) técnica, la mayoría de trabajos utilizaron la encuesta (71 informes, $26 \%$ aproximadamente), esta característica es predecible debido a la importancia de este instrumento en las Ciencias Sociales; seguida de la observación (55 informes, 20\% aproximadamente) que es una técnica muy utilizada para describir y analizar el comportamiento. Prosiguen los informes que utilizan la revisión documental (31 informes, $11 \%$ aproximadamente); las herramientas de gestión (29 informes, 11\% aproximadamente) y también hay un número de informes que utilizaron la contrastación con estándares (28 informes, $10 \%$ aproximadamente) y la entrevista (23 informes, $8 \%$ aproximadamente) entre los más significativos. Pocos informes aplicaron el experimento, análisis estadístico, lista de cotejo o análisis bibliométrico (2 informes, 1\% aproximadamente por cada variable). Asimismo, muchos de ellos, infirieron sus resultados en función a su experiencia profesional.

Gráfico 7

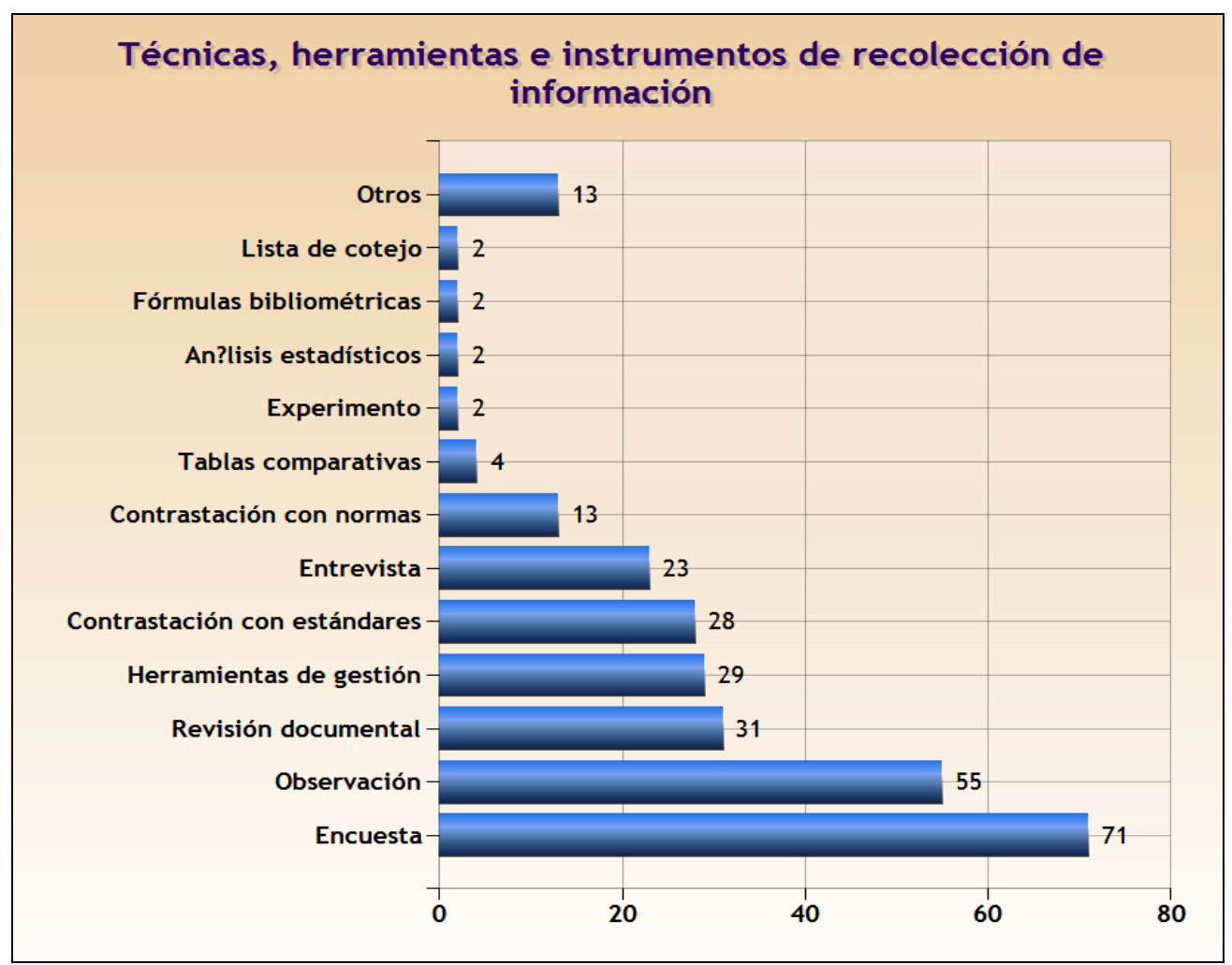

Fuente: Elaboración propia 


\section{Discusión}

Es necesario mencionar que lo más complicado en la investigación ha sido tratar de ubicar a cada uno de los estudiantes egresados de la universidad para preguntar acerca de la aplicación práctica de cada una de las propuestas planteadas en sus tesis e informes profesionales.

Hubiera sido importante estudiar los trabajos realizados en la Universidad Católica del Perú (la otra universidad que imparte estudios en la especialidad), pero por limitaciones de tiempo y de acceso a la información, solo se ha tomado a la Universidad Nacional Mayor de San Marcos. Esto significa que las conclusiones que se obtengan solo pueden referirse a los estudiantes egresados de la Universidad Nacional Mayor de San Marcos.

De todas formas, aunque hubo un gran esfuerzo por localizar a cada uno de los estudiantes egresados, no todos han sido consultados, sin embargo, no quita al presente trabajo la utilidad de los datos obtenidos, porque reflejan el estado de la investigación de los egresados de la Universidad. Hubiese sido más completo e integral analizar los artículos de revistas académicas producidos en este periodo, estudios que realizan periódicamente en otros países (Bufrem et al., 2007). Sin embargo, por razones de tiempo y acceso solo se analizaron los informes profesionales.

Actualmente el nivel de los informes profesionales ha mejorado, tanto en el aspecto metodológico, temático y de forma, ha mejorado significativamente, lo que es un indicador positivo para la EAPBCI y se orienta por buen camino.

\section{Conclusiones}

a) La investigación en la Escuela de Bibliotecología de la Universidad Nacional Mayor de San Marcos ha mejorado en los últimos años, no solo en cantidad, sino en la calidad de los trabajos de investigación, ya que cada vez tienen más alcance y se utilizan técnicas e instrumentos para la recolección de datos más válidos, sin embargo es casi nula la elaboración de tesis debido a la facilidad que dan los seminarios de titulación de la escuela.

b) tipos de unidades donde se ha realizado el mayor número de investigaciones son las bibliotecas universitarias y especializadas, debido principalmente a la tendencia a la acreditación de este tipo de unidades, así como los nuevos servicios. Por otro lado, los estudios en la Biblioteca Nacional y los Archivos, este último aspecto importante a considerar, pues esto es un buen indicador del mercado laboral, ya que los informes son experiencias profesionales, siendo los archivos el lugar donde se encuentran trabajando muchos de nuestros egresados.

c) Los temas en donde más investigan los egresados son Servicios bibliotecarios, seguido de lejos por Administración de Unidades de Información y Evaluación de unidades de información. Son nulas las 
investigaciones acerca de la historia del libro o de la profesión y pocos investigan acerca de aspectos legales y bibliometria.

d)La gran mayoría de los estudios son descriptivos y las técnicas e instrumentos de recolección de la información más utilizados son prioritariamente las encuestas, así como la observación. Se utiliza, también, la entrevista y la revisión documental. Debido a la naturaleza de la especialidad, también se ha utilizado el análisis bibliométrico (Ley de Bradford y Ley de Lotka), fórmulas matemáticas y estadísticas, contrastación con modelos de acreditación, cabe recalcar que la evaluación realizada a través de la contrastación con normas y estándares tiene una alta preferencia.

e)Solo un $14 \%$ de los egresados ha llevado a cabo su propuesta en la práctica en una unidad de información real, aspecto importante a considerar en el futuro, pues cuando se realiza una investigación para la obtención de un grado o para la licenciatura existe una doble satisfacción para el aspirante la consecución del título y el aporte a la especialidad, ya sea teórico o práctico.

f)Los lineamientos para reorientar la investigación en la Escuela de Bibliotecología y Ciencias de la Información, según la información recopilada y después de analizar los datos obtenidos, son los siguientes:

i)La Escuela de Bibliotecología debería tener un banco de temas de investigación pertinentes a la solución de problemas específicos, con aportes multidisciplinarios a nivel descriptivo y exploratorio, que cubran vacíos teóricos y prácticos de la especialidad, tanto para tesis como informes profesionales.

ii)Se deberían realizar gestiones de sensibilización para que las empresas y diferentes organizaciones del Estado privilegien a los egresados que se titulen por la modalidad de tesis a la hora de ser seleccionados en la actividad laboral, ya que un egresado que ha elaborado una tesis tiene más dominio de conocimientos, metodología para elaborar cualquier trabajo, mayor análisis para cualquier situación que se le presente en la práctica y, sobretodo, es más perseverante y minucioso, características que repercutirán, sin lugar a dudas, en su vida laboral.

iii)Es necesario ser más exigente en la utilización de instrumentos y herramientas validadas en los informes profesionales a efecto de que los resultados que se obtengan en estos trabajos se puedan generalizar. Los trabajos de los egresados, no solo deben ser un aporte práctico hacia la institución donde laboran, sino deben tener un aporte teórico y sobretodo metodológico que permita a la especialidad tener un cuerpo de conocimientos que la identifiquen, teniendo en cuenta la multidisciplinariedad de la especialidad.

iv)En las jornadas curriculares es necesario que los cursos de metodología no solo se orienten a la elaboración de un trabajo final de investigación, sino también a la formación de investigadores y a la divulgación científica. Se debe orientar a leer investigaciones ya consumadas, como decía Piscoya (2007) él que quiere ser investigador, 
tiene que leer investigaciones. Se deben realizar talleres de formulación de problemas, operacionalizar variables, elaboración de matrices de consistencia, validar instrumentos, utilización de herramientas estadísticas, esto último, que es de lo que acaecemos en nuestra especialidad.

v)La EAPBCI debería fortalecer vínculos con otras instituciones vinculadas a la investigación como la Dirección de Investigaciones Bibliotecológicas de la Biblioteca Nacional del Perú, el Colegio de Bibliotecólogos, la Pontificia Universidad Católica del Perú, para aunar recursos para lograr darle sentido a la actividad investigativa de la profesión.

\section{Referencias}

ALFARO JIMENEZ, S. Competencias profesionales de los recursos humanos de las bibliotecas universitarias del Perú. Escuela Académico Profesional de Bibliotecología y Ciencias de la Información. Lima: Universidad Nacional Mayor de San Marcos. Facultad de Letras y Ciencias Humanas, 2011. 171p.

BIBLIOTECA NACIONAL DEL PERÚ. La investigación y la docencia bibliotecológica en el Perú: ponencias y conclusiones. Lima,19 al 20 de noviembre 2002. Lima: Biblioteca Nacional del Perú, 2003.

BIBLIOTECA NACIONAL DEL PERÚ. Avances, logros y desafíos de la Investigación Bibliotecológica. In: ENCUENTRO DE INVESTIGADORES Y DOCENTES EN EL ÁREA DE BIBLIOTECOLOGÍA Y CIENCIAS DE LA INFORMACIÓN, 2., 2009, Lima. [Annalles...]. Lima: Biblioteca Nacional del Perú, 2009. 217p.

BUFREM, L. S., SILVA, H. D. F. N., RAMOS, C. L. S., FABIAN, M. \& SORRIBAS, T. V. Produção científica em ciência da informação: análise temática em artigos de revistas brasileiras. Perspectivas em Ciência da Informação, Belo Horizonte, v. 12, p. 38-49, 2007.

CORZO C., O. Investigación bibliotecológica universitaria en el Perú. In: BIBLIOTECA NACIONAL DEL PERÚ. La investigación y la docencia bibliotecológica en el Perú: ponencias y conclusiones. Lima, 2002). p. 165-175.

CORZO, O.; CHÁVEZ SÁNCHEZ, H.; ESTRADA-CUZCANO, A.; LUMBRERAS H.; S.; ROMERO, B.. La Bibliotecología y Ciencias de la Información en el Perú. Claustro Pleno, p. 29-63, 1986.

ESTRADA-CUZCANO, A.; CHÁVEZ-SÁNCHEZ, H.; CHÁVEZ-PALOMINO, L.M.. Análisis de la producción científica en la Facultad de Letras y Ciencias Humanas de la UNMSM. In: PEÑA, V. A.; RIVERA, P. H. ; SCHULZ, V; TÁPIA, \& R. B. (Ed.) La producción científica en San Marcos: Hechos, cifras y estándares internacionales (2002 - 2010). Lima: UNMSM, 2011. p. 571-579. 
ESTRADA-CUZCANO, A.; HUAMAN-HURIARTE, R. Posicionamiento de las bibliotecas universitarias peruanas en los medios de comunicación. Investigación Bibliotecológica, México, DF, 24, 13-41, 2010.

MIRANDA MERUVIA, I. La Escuela Académico-Profesional de Bibliotecología y Ciencias de la Información de la Universidad Nacional Mayor de San Marcos, Perú: recuento y perspectivas. In: SEMINARIO INFOBILA: como apoyo a la investigación y educación en Bibliotecología en América Latina y el Caribe. Editores: F. F. MARTÍNEZ ARELLANO; J. J. CALVA GONZÁlEZ. Memória... México, DF: UNAM: CUIB, 2005. p. 210227.

PEÑA, V. A.; RIVERA, P. H.; SCHULZ, P. A. B.; TÁPIA, J. R. B.. La producción científica en San Marcos: Hechos, cifras y estándares internacionales (2002-2010), Lima: UNMSM, 2011.

QUIROZ DE GARCÍA, R. La investigación en la Universidad Mayor de San Marcos. In: BIBLIOTECA NACIONAL DEL PERÚ. La investigación y la docencia bibliotecológica en el Perú: ponencias y conclusiones. Lima: Biblioteca Nacional del Perú, 2002. p. 155-164.

QUISPE GERÓNIMO, C. Validación de Open - Access para la investigación bibliotecológica. In: ENCUENTRO DE INVESTIGADORES Y DOCENTES EN EL ÁREA DE BIBLIOTECOLOGÍA Y CIENCIAS DE LA INFORMACIÓN, 2., 2009, Lima. [Memória...]. Lima: Biblioteca Nacional del Perú, 2009. p. 6781.

SILVA SANTISTEBAN CEVALLOS, T. Indice de tesis de la Escuela Nacional de Bibliotecarios, Lima: Biblioteca Nacional del Perú, 1984.

VÍLCHEZ ROMÁN, C. La investigación bibliotecológica en el Perú. La investigación bibliotecológica en las universidades peruanas: período 1990 - 2003. Lima: Biblioteca Nacional del Perú, 2005. p. 24-32. 